\title{
Uso do óleo essencial derivado da Alpinia Zerumbet na espasticidade decorrente de lesão nervosa central - Uma revisão sistemática
}

\author{
Patrícia Caroline de Oliveira Lencina ${ }^{\mathrm{a}}$, Andriele Gonçalves Vilaverde ${ }^{\mathrm{a}}$, André Luís \\ Ferreira Meireles ${ }^{\mathrm{a}, \mathrm{b} *}$
}

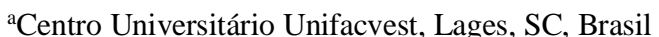

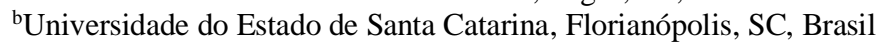

\section{Histórico do Artigo: Recebido em 20/04/2020 \\ Aceito em 03/09/2020}

\section{Palavras-chave:}

Medicamentos fitoterápicos; hipertonia muscular; fisioterapia; paralisia cerebral

Keywords: Phytotherapeutic drugs; muscle hypertonia; physiotherapy; cerebral palsy.

\begin{abstract}
RESUMO
A espasticidade é um fenômeno que ocorrerá devido a lesão no neurônio motor superior, e pode ser definida como uma hiperexcitabilidade do reflexo de estiramento. Recentemente um óleo essencial derivado de uma planta conhecida como Alpinia Zerumbet, e comercializado como fitoterápico, vem sendo usado para modular a espasticidade causada por doenças neurológicas como o Acidente Vascular Encefálico (AVE) e Paralisia Cerebral (PC). O objetivo desse estudo foi sumarizar as evidências sobre os efeitos do óleo essencial derivado da Alpinia Zerumbet (OAZ) em pacientes com espasticidade. Trata-se de uma revisão sistemática. Artigos que envolvessem o uso do OAZ no tratamento da espasticidade em doenças neurológicas foram pesquisados nas seguintes bases de dados: Scielo, Lilacs, Pubmed, PEDro, EMBASE e Google Acadêmico. Estudos que investigassem a ação do OAZ sobre lesões nervosas periféricas ou condições não neurológicas, estudos de revisão e estudos pré-clínicos, foram excluídos desse estudo. A escala PEDro e a ferramenta Quality assessment tool for before-after (pre-post) studies with no control group foram utilizadas para avaliação metodológica dos estudos. Cinco estudos foram selecionados seguindo os critérios de inclusão. Desses, dois ensaios clínicos controlados randomizados, dois ensaios clínicos quase-experimentais e um era uma série de casos. As populações estudadas nesses artigos foram AVE, PC e lesão medular, e os principais desfechos avaliados foram espasticidade e função motora. A administração do OAZ associada a cinesioterapia foi capaz de diminuir a espasticidade muscular e melhorar função de pacientes neurológicos. Entretanto, devido a fragilidade metodológica encontrada nas atuais evidências, ainda não é possível atestar sua eficácia.
\end{abstract}

Application of Alpinia Zerumbet essential oil in spasticity after central nervous injury - A systematic review

\section{ABSTRACT}

The spasticity occurs due to lesion in upper motor neuron and could be defined as a hyperexcitability of the stretch reflex. Recently an essential oil extracted from a plant known as Alpinia Zerumbet, and marketed as an herbal medicine, it has been used to modulate spasticity caused by neurological diseases such as Stroke and Cerebral Palsy (CP). The objective was to summarize the evidence about the effects of Alpinia Zerumbet essential oil (OAZ) in patients with spasticity. It is a systematic review study. Studies exploring the use of OAZ in spasticity managment of neurological diseases were searched in the following databases: Scielo, Lilacs, Pubmed, Science Direct, PEDro, EMBASE e Google Academic. Studies exploring the action of OAZ in peripheral nerve injuries or in non-neurological conditions, review studies and basic studies, were excluded. PEDro scale and Quality assessment tool for before-after (pre-post) studies with no control group tool were used to assess the quality of methodology in the studies. Results: Five studies were selected following the include criteria. Of these, there were two were clinical randomized trials, two were quasi-experimental trials, and the last one it was a case series study. Stroke, CP and spinal cord injury patients were the studied population, and the mainly outcomes evaluated were muscular spasticity and motor function. The administration of OAZ associated with kinesiotherapy was able to decrease the muscular spasticity and improve the motor function in neurological patients. However, due to the methodological weaknesses founded in actual evidence, it is not yet possible to attest to its effectiveness.

\footnotetext{
* Autor correspondente: meireles.alf@gmail.com (Meireles A.L.F)
} 
P. C. de O. Lencina et al. / Vittalle v. 32, n. 3 (2020) 214-224

\section{Introdução}

A espasticidade é uma desordem sensório-motora, caracterizada por uma hiperexcitabilidade do reflexo de estiramento muscular, causada por lesão nervosa central (1). A espasticidade é uma das principais causas da perda ou incapacidade da função motora, e é comumente observada em pacientes com Acidente Vascular Encefálico (AVE), Paralisia Cerebral (PC), trauma raquimedular e traumas cranioencefálicos, todas essas condições apresentam uma alta incidência (1-3).

Atualmente o tratamento da espasticidade geralmente é feito por uma associação entre intervenções farmacológicas e não-farmacológicas. $\mathrm{O}$ tratamento farmacológico pode ser composto da administração de fármacos como o Bacoflen, Benzodiazepínicos e Gabapentinas, que atuarão nos receptores ácido gama-aminobutírico (GABA) resultando, de forma generalista, na depressão do sistema nervoso central, causando uma diminuição de tônus muscular de forma global (4-6). Outra vertente farmacológica é a administração de bloqueadores neurolíticos, toxina botulínica e fenol, que promoverão inibição da liberação de acetilcolina vesicular dos terminais do nervo présináptico na junção neuromuscular e neurólise química no nervo do músculo espástico, respectivamente $(4,7)$. Ambas as opções podem causar efeitos adversos importantes para a saúde do paciente. Os principais efeitos adversos da administração da toxina botulínica é o desenvolvimento de imunoresistência e migração da toxina para outras regiões do corpo, e as da administração do fenol são dor neuropática e alterações vesicais (7).

O tratamento não-farmacológico mais utilizado para o tratamento da espasticidade é realizado através de intervenções disponíveis pela fisioterapia com o intuito de proporcionar melhores níveis de função e maior independência, entre eles podemos citar a equoterapia, Conceito Neuroevolutivo Bobath e terapia de vibração corporal (5). Tais escolhas terapêuticas, farmacológicas ou não, irão variar em relação a sua efetividade de paciente para paciente, mas há o consenso de que devem ser sinérgicas para melhor otimização do quadro geral do paciente $(5,8)$.

Desde o ano de 2017 um novo agente terapêutico, Ziclague ${ }^{\circledR}$ (Alpinia zerumbet Zingiberaceae, Hebron - Brasil), vem sendo utilizado na prática clínica por fisioterapeutas brasileiros para a modulação da espasticidade decorrente de lesões no sistema nervoso central. Esse fitoterápico é composto de óleos essenciais derivados de uma planta chamada Alpinia Zerumbet, comumente encontrada na Região Nordeste do Brasil (9-11). Esse fitoterápico é comercializado em forma de spray e, segundo a bula do fabricante, após 15 minutos da aplicação sobre a pele correspondente à musculatura espástica de pacientes pós-lesão em neurônio motor superior, proporciona uma diminuição temporária da espasticidade facilitando a atuação fisioterapêutica $(11,12)$.

Estudos pré-clínicos reportam que a aplicação do óleo essencial derivado da Alpinia Zerumbet (OAZ) tem um efeito antiespasmódico e miorrelaxante na musculatura lisa do íleo de coelhos e na musculatura cardíaca de ratos, e que esses efeitos estariam relacionados com a inibição dos canais $\mathrm{Ca} 2+$ tipo $\mathrm{L}$ e com a competição pós-sináptica de acetilcolina no músculo, causado por componente presente nesse óleo, o terpereno (Terpieno-4-ol e 1,8-cineol) (13,14). Estudo investigando a ação 1,8-cineol no músculo cardíaco, demonstrou que esse composto foi capaz de diminuir a força de contração dos músculos papilares de ratos (15). Além desses mudanças geradas pela ação do óleo e seus componentes, Leal-Cardoso et al. (16) observaram que a aplicação desse óleo diminui o potencial de ação do nervo ciático de ratos.

Em contrapartida, há estudos clínicos apresentando efeitos antiespasmódicos em músculos espásticos de humanos. Cândido et al. (17) demonstraram que a associação do 
OAZ com 10 sessões de fisioterapia em pacientes com AVE e lesão medular, diminuiu a espasticidade e o potencial evocado muscular em membros inferiores. Maia et al. (18), em ensaio quase-experimental, observou que a aplicação do óleo no músculo gastrocnêmio do lado afetado em pacientes pós-AVE, diminuiu a espasticidade e o potencial evocado.

Tendo em vista a possível potencialidade terapêutica desse fitoterápico na modulação da espasticidade decorrente de lesões corticais comuns como a PC e o AVE, essa revisão tem como objetivo sumarizar os efeitos da administração do OAZ sobre a espasticidade muscular, de forma isolada ou agindo como terapia coadjuvante, em lesões no sistema nervoso central.

\section{Material e métodos}

Essa revisão sistemática foi realizada utilizando-se as orientações do Preferred Reporting Items for Systematic Reviews and Meta-Analyses (PRISMA) e levando em consideração outros tipos de estudo, além dos ensaios clínicos controlados e randomizados (19).

\section{Critérios de Elegibilidade}

Foram incluídos nessa revisão: ensaios clínicos controlados randomizados ou não, estudo quase-experimentais e relatos de caso, nos idiomas português e inglês, que avaliaram os efeitos da aplicação do OAZ de forma isolada ou coadjuvante a outro tratamento em pacientes com espasticidade decorrente de lesão no sistema nervoso central, sem limite de idade e de ambos os sexos. Estudos que explorassem os efeitos da aplicação do OAZ em outros aspectos musculares (ex.: força muscular, atividade elétrica) de paciente pós lesão neural, também foram incluídos.

Estudos pré-clínicos, estudos clínicos que investigassem a ação do OAZ sobre lesões nervosas periféricas ou condições não neurológicas, estudos do tipo revisão e estudos em outros idiomas que não fossem o português ou inglês, ou que não atendessem aos critérios de elegibilidade pré-estabelecidos foram excluídos dessa revisão.

\section{Estratégia de Busca}

A busca pelos artigos dessa revisão foi realizada de outubro a dezembro de 2018 nas seguintes bases de dados e repositórios: Scientific Electronic Library Online (SciELO), Google Scholar, PubMed, Literatura Latino-americana e do Caribe em Ciências da Saúde (LILACS), EMBASE e Physiotherapy Evidence Database (PEDro). Os termos sobre a intervenção: alpinia zerumbet, óleo essencial e ziclague, foram pesquisadas sozinhas e associadas aos descritores da população, doenças ou desfecho: paralisia cerebral, encefalopatia crônica não progressiva da infância, acidente vascular cerebral, acidente vascular encefálico, traumatismo raquimedular, lesão medular, Parkinson, doença de Parkinson, traumatismo cranioencefálico, traumatismo craniano, com o uso de operadores booleanos E e OU, dependendo da base de dados utilizada.

\section{Etapas da Busca}

Todos os passos dessa revisão foram conduzidos por dois pesquisadores independentes (P.C.O.L. e A.G.V.), previamente treinados, e que ao final de cada passo se reuniram para estabelecimento do consenso de cada etapa. Quando houve discordância em 
alguma fase do processo, a decisão foi tomada por um terceiro pesquisador (A.L.F.M.). A busca pelos artigos que compuseram essa revisão foi o primeiro passo desse estudo. No segundo passo, os títulos de todos os estudos encontrados nas bases de dados eletrônicas foram avaliados e os que não atendiam aos critérios de inclusão foram excluídos. O mesmo procedimento foi utilizado na terceira etapa, com a leitura e análise do resumo dos estudos incluídos na segunda etapa. Na quarta etapa foi realizada a leitura de todos os textos na íntegra, incluídos nas fases anteriores e aqueles que não se encaixaram nos critérios de inclusão do estudo, foram excluídos. Na quinta etapa foi realizada busca manual ativa na lista de referências de todos os trabalhos incluídos após busca nas bases de dados eletrônicas, seguindo os mesmos procedimentos anteriores. Após a leitura completa dos artigos selecionados nessa revisão, as informações de interesse e os critérios necessários para avaliação desses estudos foram coletadas e registradas.

A escala PEDro foi aplicada nos ensaios clínicos controlados aleatorizados incluídos nessa revisão. Essa ferramenta é usada para avaliação da qualidade metodológica de artigos científicos do tipo ensaio controlados aleatorizados, compostas de 11 critérios, tendo escore variando de 1 a 10, quanto maior o valor, mais bem classificado o artigo estará. Aqui, optamos por utilizar a seguinte classificação para mensurar a nota final da escala PEDro: escore de 10 a 6 (alta qualidade); 5-4: (média qualidade) e 0-3 (baixa qualidade) (20).

Para avaliar a qualidade metodológica dos estudos quase-experimentais, a ferramenta Quality assessment tool for before-after (pre-post) studies with no control group foi aplicada. Esta ferramenta é composta por 12 critérios que compreendem cinco possibilidades de respostas: Sim, não, não determinado, não relatado e não aplicável. A partir do resultado, a qualidade metodológica do artigo é mensurada como boa (escore maior que 6), razoável (entre 6 e 4) ou ruim (abaixo de 4) (21).

\section{Resultados}

Foram encontrados 165 artigos no início do processo de pesquisa nas bases de dados. Após a análise de títulos, leitura de resumos, leitura de textos na íntegra e busca manual por lista de referências, 5 artigos se encaixaram nos critérios de elegibilidade e foram selecionados para a presente revisão (Figura 1). As principais características dos estudos selecionados estão dispostas na Tabela 1. 
Vittalle - Revista de Ciências da Saúde v. 32, n. 3 (2020) 214-224

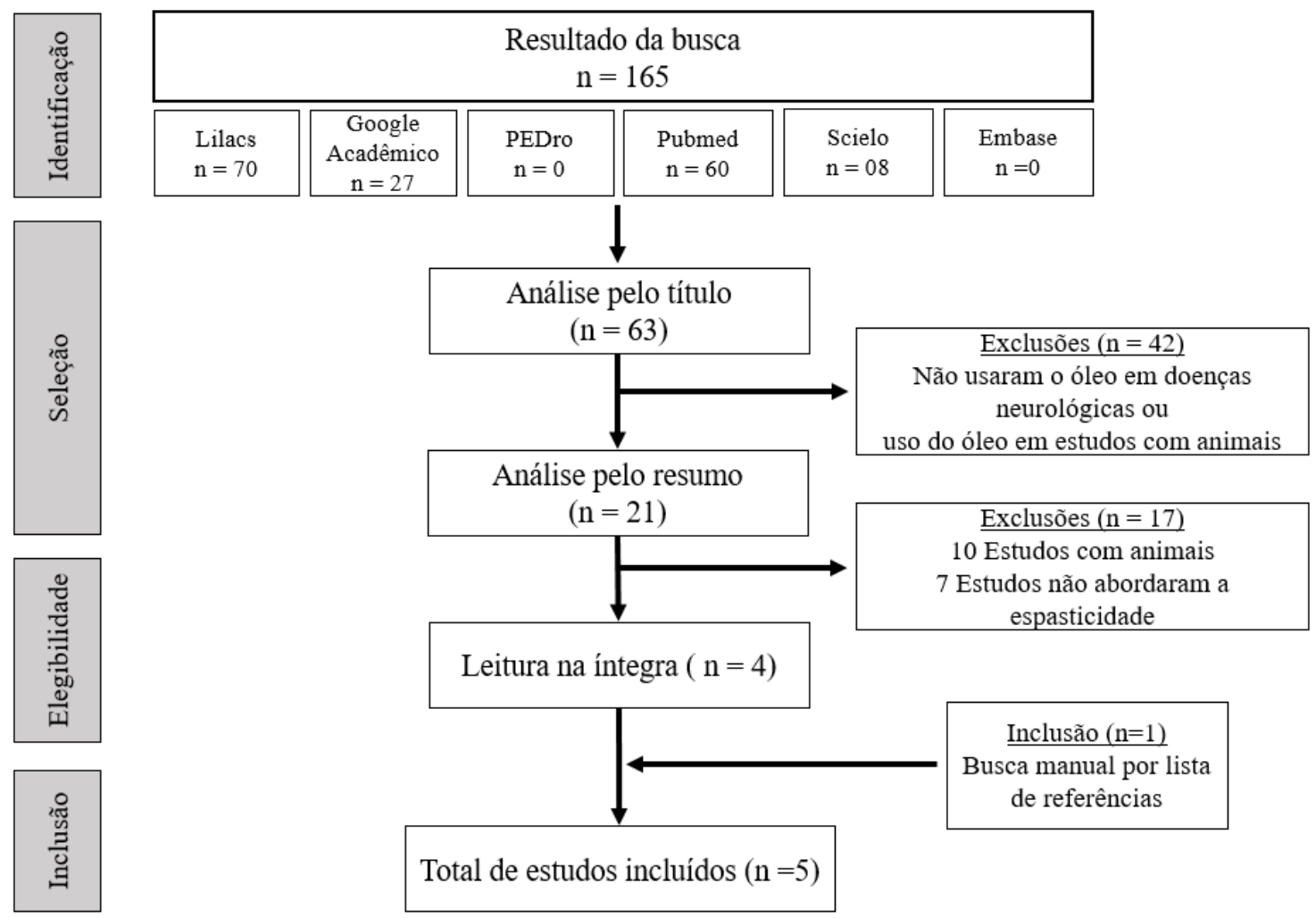

Figura 1 - Fluxograma com as etapas realizadas para seleção de artigos que compuseram essa revisão.

Tabela 1 - Caracterização dos estudos quanto o tipo de estudo, $\mathrm{n}$ amostral, intervenções e resultados principais.

\begin{tabular}{|c|c|c|c|c|}
\hline $\begin{array}{c}\text { Autor/ } \\
\text { Ano }\end{array}$ & $\begin{array}{l}\text { Tipo de } \\
\text { Estudo }\end{array}$ & $\mathbf{N}$ & População/Intervenção & Resultados Principais \\
\hline $\begin{array}{l}\text { Oliveira } \\
\text { et al. (22) }\end{array}$ & $\begin{array}{c}\text { Quase- } \\
\text { experimental }\end{array}$ & 20 & $\begin{array}{l}\text { Pacientes com LM } \\
10 \text { sessões de cinesioterapia associada } \\
\text { a aplicação do OAZ. }\end{array}$ & $\begin{array}{l}\text { \#Melhora da independência funcional (Enviro } \\
\text { FIM - motor tasks e diminuição de } \\
\text { espasticidade (Asworth scale). }\end{array}$ \\
\hline $\begin{array}{l}\text { Cândido } \\
\text { et al. (17) }\end{array}$ & ECR & 50 & $\begin{array}{l}\text { Pacientes com LM e AVE. } \\
\text { GC: } 10 \text { sessões de cinesioterapia } \\
\text { associada aplicação de óleo vegetal } \\
\text { GI: } 10 \text { sessões de cinesioterapia } \\
\text { associada a aplicação do OAZ. }\end{array}$ & $\begin{array}{l}\text { "A cinesioterapia associada ao OAZ diminuiu a } \\
\text { espasticidade (Modified modified Ashworth } \\
\text { scale) no GI: }(2,54 \pm 0,62 \text { vs. } 1,19 \pm 0,11 \text {; } \\
\text { p }<0,001) \text { e melhorou o recrutamento muscular } \\
\text { (Eletromiografia de superfície) no GI: }(62,04 \pm \\
22,72 \text { vs. } 70,87 \pm 24,40 ; \mathrm{p}<0,05) \text {. }\end{array}$ \\
\hline $\begin{array}{l}\text { Cândido } \\
\text { et al. (23) }\end{array}$ & Série de Casos & 8 & $\begin{array}{l}\text { Crianças e adolescentes com PC, e um } \\
\text { adulto com AVE. } \\
\text { Submetidos a associação de } \\
\text { cinesioterapia e aplicação do OAZ, em } \\
\text { diferentes tempos de tratamento } \\
\text { subagudo (11 a } 35 \text { aplicações do } \\
\text { OAZ) e (crônico } 36 \text { a } 72 \text { aplicações do } \\
\text { OAZ) entre } 2 \text { meses e } 6 \text { meses de } \\
\text { tratamento. }\end{array}$ & $\begin{array}{l}\text { \#A associação de cinesioterapia e o OAZ } \\
\text { proporcionou uma diminuição da espasticidade } \\
\text { (Modified modified Ashworth scale) nos } \\
\text { diferentes casos avaliados e nos diferentes } \\
\text { tempos. }\end{array}$ \\
\hline $\begin{array}{l}\text { Maia et } \\
\text { al. (18) }\end{array}$ & $\begin{array}{c}\text { Quase- } \\
\text { experimental }\end{array}$ & 15 & $\begin{array}{l}\text { Pacientes diagnosticados com AVE. } \\
10 \text { aplicações do OAZ nos } \\
\text { gastrocnêmios (ventre lateral e } \\
\text { medial) do lado afetado e saudável. }\end{array}$ & $\begin{array}{l}\text { A administração do OAZ nos gastrocnêmios } \\
\text { promoveu um aumento nos valores } \\
\text { eletromiográficos da root mean square no lado } \\
\text { afetado: }(99,18 \pm 45,26 \text { vs. } 108,01 \pm 47,63 ; \mathrm{p}= \\
0,002 \text {. (ventre lateral)) e }(76,09 \pm 54,64 \text { vs. } \\
101,46 \pm 86,84 ; \mathrm{p}=0,0012 \text {. (ventre medial)) e } \\
\text { na medida median power frequency }(71,97 \pm \\
30,00 \text { vs. } 80,37 \pm 36,30 ; \mathrm{p}=0,0003 \text {. (ventre } \\
\text { lateral)) e }(55,63 \pm 40,39 \text { vs. } 74,51 \pm 62,70 ; \mathrm{p}= \\
\text { 0,0003. (ventre medial)). Segundo os autores, o } \\
\text { protocolo utilizado foi capar de afetar a } \\
\text { contractilidade do músculo espástico, gerando } \\
\text { relaxamento muscular e aumentando a } \\
\text { performance contráctil nos gastrocnêmios } \\
\text { nessa população. }\end{array}$ \\
\hline
\end{tabular}


Tabela 3 - Ferramenta de avalição da qualidade metodológica Quality assessment tool for before-after (pre-post) studies with no control group.

\begin{tabular}{|c|c|c|}
\hline $\begin{array}{l}\text { Ferramenta de avaliação da qualidade para estudos antes e depois } \\
\text { (pré-pós) sem grupo controle }\end{array}$ & $\begin{array}{l}\text { Oliveira et } \\
\text { al. (22) }\end{array}$ & $\begin{array}{l}\text { Maia et } \\
\text { al. (18) }\end{array}$ \\
\hline 1. A pergunta ou objetivo do estudo foi claramente reportado? & $\mathrm{S}$ & $\mathrm{S}$ \\
\hline $\begin{array}{l}\text { 2. Os critérios de elegibilidade/seleção para a população do estudo foram } \\
\text { pré-especificados e claramente descritos? }\end{array}$ & $\mathrm{S}$ & $\mathrm{S}$ \\
\hline $\begin{array}{l}\text { 3. Os participantes do estudo eram representativos daqueles que seriam } \\
\text { elegíveis para o teste / serviço / intervenção na população geral ou clínica } \\
\text { de interesse? }\end{array}$ & $\mathrm{S}$ & $S$ \\
\hline $\begin{array}{l}\text { 4. Todos os participantes elegíveis que atenderam aos critérios de } \\
\text { inscrição pré-especificados foram inscritos? }\end{array}$ & S & S \\
\hline $\begin{array}{l}\text { 5. O tamanho da amostra era suficientemente grande para fornecer } \\
\text { confiança nas descobertas? }\end{array}$ & $\mathrm{N}$ & $\mathrm{N}$ \\
\hline $\begin{array}{l}\text { 6. O teste / serviço / intervenção foi claramente descrito e realizado de } \\
\text { forma consistente em toda a população do estudo? }\end{array}$ & $\mathrm{N}$ & $S$ \\
\hline $\begin{array}{l}\text { 7. As medidas de resultado foram pré-especificadas, claramente definidas, } \\
\text { válidas, confiáveis e avaliadas de forma consistente em todos os } \\
\text { participantes do estudo? }\end{array}$ & $S$ & $S$ \\
\hline $\begin{array}{l}\text { 8. As pessoas que avaliavam os resultados estavam cegas às exposições / } \\
\text { intervenções dos participantes? }\end{array}$ & NA & NA \\
\hline $\begin{array}{l}\text { 9. A perda de acompanhamento após a linha de base foi de } 20 \% \text { ou } \\
\text { menos? Os perdidos no acompanhamento foram contabilizados na análise? }\end{array}$ & NA & NA \\
\hline $\begin{array}{l}\text { 10. Os métodos estatísticos examinaram as mudanças nas medidas de } \\
\text { resultados antes e depois da intervenção? Foram realizados testes } \\
\text { estatísticos que forneceram valores de p para as alterações pré e pós? }\end{array}$ & $S$ & S \\
\hline $\begin{array}{l}\text { 11. As medidas de resultado de interesse foram tomadas várias vezes antes } \\
\text { da intervenção e várias vezes após a intervenção (ou seja, elas usaram um } \\
\text { desenho de séries temporais interrompidas)? }\end{array}$ & $\mathrm{N}$ & $\mathrm{N}$ \\
\hline $\begin{array}{l}\text { 12. Se a intervenção foi conduzida em nível de grupo (por exemplo, um } \\
\text { hospital inteiro, uma comunidade etc.), a análise estatística levou em } \\
\text { consideração o uso de dados em nível individual para determinar os efeitos } \\
\text { em nível de grupo? }\end{array}$ & NR & NR \\
\hline TOTAL & 6 & 7 \\
\hline
\end{tabular}

Legendas: ND, não determinado; NA, não aplicável; NR, não reportado; S, sim; N, não.

\section{Discussão}

Essa revisão se propôs a avaliar os efeitos da administração do OAZ sobre a modulação da espasticidade pós-lesão em sistema nervoso central. Dos cinco artigos incluídos nessa revisão, quatro usaram o óleo essencial como terapia coadjuvante a fisioterapia, e um único estudo avaliou o seu uso exclusivo na atividade elétrica muscular por meio da eletromiografia de superfície.

Maia et al. (18) investigaram o efeito da administração do OAZ sobre a atividade muscular dos gastrocnêmios de vinte pacientes pós AVE com o uso de eletromiografia de superfície. Para tal observação, os autores além de compararem o antes e depois de 10 aplicações diárias do fitoterápico por via dérmica no músculo gastrocnêmio do lado afetado, também avaliaram a atividade elétrica do gastrocnêmio do lado sadio desses pacientes. Os autores relataram que a aplicação diária do óleo nos músculos afetados desses pacientes promoveu diferenças estatisticamente significativas em relação a Root Mean Square (RMS) na eletromiografia de superfície - Médias \pm desvio padrão: (de 99,18 $\pm 45,26$ para 108,01 $\pm 47,63 ;(\mathrm{p}=0,002)$ ventre lateral) e (de 76,09 $\pm 54,64$ para $101,46 \pm 86,84 ;(\mathrm{p}=0,0012)$ ventre medial), e na medida median power frequency ( $\mathrm{de}$ $71,97 \pm 30,00$ para 80,37 $\pm 36,30 ;(p=0,0003)$ ventre lateral $)$ e (de 55,63 \pm 40,39 para $74,51 \pm 62,70(p=0,0003))$; ventre medial). Assim, as 10 aplicações do óleo foram 
capazes de afetar a contractilidade do músculo espástico, gerando relaxamento muscular e aumentando a performance contráctil nos gastrocnêmios nessa população.

Além de Maia, et al. (18), outros dois estudos investigaram os efeitos do uso da administração do OAZ em pacientes com AVE. Cândido et al. (17) em ensaio clínico controlado randomizado (ECR) estudaram os efeitos desse óleo associado à cinesioterapia, sobre a espasticidade (Modification of the Modified Ashworth Scale $M M A S$ ), recrutamento muscular (eletromiografia de superfície), e também verificaram a pressão arterial de pacientes sobreviventes de AVE e lesão medular. Para tal, 50 pacientes foram separados entre grupo tratado com a fisioterapia mais administração por via dérmica do OAZ $(n=30)$, e um grupo controle submetido à fisioterapia mais o uso de óleo de girassol $(n=20)$. Esses grupos foram avaliados antes e após 10 sessões, com duração de $1 \mathrm{~h}$ em dias alternados. Por razão não descrita no artigo, os autores não reportaram os valores do grupo controle antes do tratamento em nenhuma das análises citadas.

$\mathrm{Na}$ avaliação da espasticidade muscular pela MMAS, os autores reportaram que o grupo que fez fisioterapia mais uso do OAZ apresentou escore inicial de $(2,54 \pm 0,62)$ e após as sessões propostas obteve escore de $(1,19 \pm 0,11)$, havendo diferença estatística entre as médias do antes e depois desse grupo $(\mathrm{p}<0,001)$. Em relação a análise da eletromiografia de superfície, antes do protocolo de atendimento e uso de óleo, os pacientes apresentaram média de RMS de $(62,04 \pm 22,72)$ que após as 10 sessões foi para $(70,87 \pm 24,4)$ sendo essas médias estatisticamente diferentes $(\mathrm{p}<0,05)$, o grupo controle após a fisioterapia apresentou média de RMS de $(131,8 \pm 22,68)$. Não houve diferenças na pressão arterial, sistólica ou diastólica, em nenhum grupo. Os autores concluem nesse estudo que a associação entre cinesioterapia e o OAZ demonstrou-se eficaz na melhoria do recrutamento muscular de pessoas com espasticidade e que provavelmente a melhor eficiência na contração muscular estaria envolvida na melhor qualidade do tônus muscular e da tensão passiva das fibras musculares. Embora a conclusão desse artigo seja animadora, alguns pontos metodológicos como ausência dos valores baseline do grupo controle e as grandes amplitudes nas medidas de dispersão nos levam a ter parcimônia com a interpretação e transposição desses resultados para a clínica.

No segundo estudo abordando os efeitos do OAZ no paciente com AVE, Cândido et al. (22) realizaram um estudo do tipo série de casos $(n=7)$ e observaram a ação coadjuvante do óleo sendo aplicado por um período subagudo (11 a 35 aplicações) e um período crônico (36 a 72 aplicações) na espasticidade de crianças e adolescentes de diferentes idades, com diagnóstico de PC, e um adulto com AVE. Usando a escala MMAS, os autores reportaram que todos os pacientes tiveram uma diminuição estatisticamente significativa em todos os casos, seja usando um protocolo mais curto ou mais longo. Os dados brutos de médias e desvios padrões não foram reportados no texto.

Em adição ao estudo de Cândido et al. (17), Oliveira et al. (22) em um estudo do tipo quase-experimental, investigaram o uso do OAZ em 20 pacientes com lesão medular. Desses pacientes, 10 apresentaram tetra ou paraplegia, e destes, 6 tinham lesão incompleta (ASIA C). Após 10 sessões de fisioterapia associadas com a administração dérmica do OAZ, os pacientes apresentaram um escore menor de espasticidade (Ashwort Scale) e maior da independência funcional (Enviro FIM - motor tasks). Dados de médias, desvio padrões e informações sobre tempo de lesão dos pacientes não foram reportadas no texto.

O último estudo incluído nessa revisão abordou os efeitos coadjuvantes da administração do OAZ por via dérmica e inalatória, exclusivamente em crianças com 
PC. Em um ECR, Cândido e Xavier-Filho (12), investigaram o tônus muscular e atividades funcionais dinâmicas e estáticas usando o Protocolo de Durigon (24). Dessa forma, 38 crianças com $\mathrm{PC}$ foram divididas em 4 grupos: $\mathrm{O}$ grupo que recebeu cinesioterapia e administração do óleo por via dérmica $(n=20)$; o seu controle, que recebeu cinesioterapia e a administração de óleo vegetal por via dérmica $(n=6)$; $O$ grupo que recebeu cinesioterapia e a inalação do óleo $(n=6)$; e o seu controle, que recebeu cinesioterapia e a inalação de soro fisiológico. Todas os grupos receberam 10 sessões de cinesioterapia em dias alternados. Tanto o grupo que recebeu o óleo por via dérmica, quanto o grupo que recebeu o óleo por via inalatória diminuíram seus escores em relação à avaliação do tônus, de 8 para 5 e de 6,5 para 4,5 , respectivamente. Com a justificativa de seus controles não terem melhorado o tônus muscular (diminuído), os autores optaram por não apresentarem os resultados dos controles em relação a atividade funcional estática e dinâmica. Da mesma forma, os valores de médias e desvio padrão não são reportados nessas duas análises. Na avaliação funcional estática, o grupo que recebeu o óleo por via dérmica apresentou melhora nas posturas sentado, 4 apoios, ajoelhado e de pé quando comparados com ele mesmo na avaliação inicial. Realizando observação das barras dos gráficos, essa melhora parece ser de um ponto para todas as posições. Por sua vez, o grupo que recebeu o óleo por via inalatória apresentou melhora nas posições sentado, semi-ajoelhado e em pé, também quando comparados com ele mesmo na avaliação inicial. Realizando observação visual das barras dos gráficos, essa melhora parece ser de um a dois pontos para todas as posições. Já na avaliação funcional dinâmica, as duas vias de administração do óleo apresentaram melhora em apenas uma das duas aditividades avaliadas pelos autores, o engatinhar. A avaliação funcional dinâmica é graduada de 0 a 2, sendo 0 não realizou a atividade, 1 realizou com padrão anormal, e 2 realizou em padrão normal e coordenado, porém com alterações qualitativas. Pudemos observar, analisando visualmente as barras dos gráficos, que ambas barras dos grupos não alcançaram o escore 1. Os autores concluem que a via dérmica para administração do OAZ foi mais eficaz que a via inalatória, gerando uma diminuição da espasticidade e aumento da função das crianças com PC.

Assim como o outro ECR incluído nessa revisão, algumas ressalvas são importantes para a interpretação desses resultados (12). Entre elas, destacamos a ferramenta utilizada e a ausência dos dados dos grupos controle. O protocolo de Duringon é uma ferramenta que apesar de validada, é pouco investigada e reportada por artigos na área de neuroreabilitação, e, além disso, os resultados obtidos, apesar de estatisticamente diferentes, aparentam não apresentar relevância clínica. A ausência dos dados dos grupos controle, como reportado no outro ECR, não nos possibilita a certeza da real eficácia desse óleo na modulação da espasticidade nesses pacientes.

Os artigos incluídos nessa revisão demonstraram que a associação da administração do OAZ com a fisioterapia parece ser benéfica para a modulação da espasticidade, melhora da ativação muscular e melhora da função de pacientes pós-lesão encefálica.

Essa revisão possui algumas limitações, como grande heterogeneidade e baixa qualidade metodológica dos artigos incluídos. Outro ponto importante que deve ser reportado é que todos os artigos incluídos nessa revisão pertencem ao mesmo grupo de pesquisa, o que nos faz questionar sobre a validade externa dos resultados apresentados.

\section{Conclusões}

Apesar dos estudos incluídos aqui indicarem um possível efeito coadjuvante benéfico da administração do OAZ à fisioterapia convencional em pacientes com espasticidade decorrente de lesão em neurônio motor superior, a frágil metodologia e outros pontos 
levantados nessa revisão impedem a conclusão de que esse tratamento seja benéfico ou tem algum efeito nesses pacientes. Estudos com maior rigor metodológico são imperativos para atestar um possível efeito benéfico da administração do óleo essencial derivado da Alpinia Zerumbet em pacientes neurológicos.

\section{Referências}

1. Trompetto C, Marinelli L, Mori L, Pelosin E, Currà A, Molfetta L, et al. Pathophysiology of spasticity: Implications for neurorehabilitation. Biomed Res Int 2014; 2014.

2. Ma VY, Chan L, Carruthers KJ. Incidence, prevalence, costs, and impact on disability of common conditions requiring rehabilitation in the united states: Stroke, spinal cord injury, traumatic brain injury, multiple sclerosis, osteoarthritis, rheumatoid arthritis, limb loss, and back pain. Arch Phys Med Rehabil [Internet] 2014; 95(5): 986-995.e1.

3. Mukherjee A, Chakravarty A. Spasticity mechanisms - for the clinician. Front Neurol. 2010; MAR(December):1-10.

4. Chang E, Ghosh N, Yanni D, Lee S, Alexandru D, Mozaffar T. A review of spasticity treatments: Pharmacological and interventional approaches. Crit Rev Phys Rehabil Med. 2013; 25(1-2): 11-22.

5. Novak I, Morgan C, Fahey M, Finch-Edmondson M, Galea C, Hines A, et al. State of the Evidence Traffic Lights 2019: Systematic Review of Interventions for Preventing and Treating Children with Cerebral Palsy. Curr Neurol Neurosci Rep 2020; 20(2).

6. Nair KPS, Marsden J. The management of spasticity in adults. BMJ 2014; 349(August): 1-10.

7. Delgado MR, Hirtz D, Aisen M, Ashwal S, Fehlings DL, McLaughlin J, et al. Practice Parameter: Pharmacologic treatment of spasticity in children and adolescents with cerebral palsy (an evidencebased review): Report of the Quality Standards Subcommittee of the American Academy of Neurology and the Practice Committee of the Child Neurology Society. Neurology 2010; 74(4): 336-43.

8. Novak I, Mcintyre S, Morgan C, Campbell L, Dark L, Morton N, et al. A systematic review of interventions for children with cerebral palsy: State of the evidence. Dev Med Child Neurol 2013; 55(10): 885-910.

9. Moura RS de, Emiliano AF, Carvalho LCRM De, Souza MA V., Guedes DC, Tano T, et al. Antihypertensive and Endothelium-Dependent. J Cardiovasc Pharmacol 2005; 46(3): 288-94.

10. Cunha GH da, Moraes MO de, Fechine FV, Frota Bezerra FAÔ, Silveira ER, Canuto KM, et al. Vasorelaxant and antihypertensive effects of methanolic fraction of the essential oil of Alpinia zerumbet. Vascul Pharmacol [Internet] 2013; 58(5-6):337-45.

11. Ziclague, Alpinia zerumbet - Zingiberaceae - partes aéreas. Marta Melissa Leite Maia. Hebron, 2017. Bula de Remédio.

12. Farias Cândido EA, Xavier-Filho L. Viabilidade do uso do óleo essencial da Alpinia zerumbet, Zingiberaceae, na otimização do tratamento fisioterapêutico em paralisia cerebral espástica. Vol. 31, Arquivos Brasileiros de Neurocirurgia: Brazilian Neurosurgery 2012; 31: 110-5.

13. Bezerra MAC, Leal-Cardoso JH, Coelho-de-Souza AN, Criddle DN, Fonteles MC. Myorelaxant and antispasmodic effects of the essential oil of Alpinia speciosa on rat ileum. Phyther Res 2000; 14(7): 549-51.

14. Santos BA, Roman-Campos D, Carvalho MS, Miranda FMF, Carneiro DC, Cavalcante PH, et al. Cardiodepressive effect elicited by the essential oil of Alpinia speciosa is related to L-type Ca $2+$ current blockade. Phytomedicine [Internet] 2011; 18(7): 539-43.

15. Soares MC, Damiani CE, Moreira CM, Stefanon I, Vassallo DV. Eucalyptol, an essential oil, reduces contractile activity in rat cardiac muscle. Braz J Med Biol Res 2005; 38: 453-61.

16. Leal-Cardoso JH, Moreira MR, Pinto Da Cruz GM, De Morais SM, Lahlou MS, Coelho-De-Souza AN. Effects of essential oil of Alpinia zerumbet on the compound action potential of the rat sciatic nerve. Phytomedicine 2004; 11(6): 549-53.

17. Cândido JF, Lopes RM de A, Fillho LX, Cândido EAF. Influence of Alpinia Zerumbet essential oil in the kinesiotherapeutic treatment of patients with syndrome pyramidal. Int J Dev Res 2017; 07(10): 15837-43.

18. Maia MON, Dantas CG, Xavier Filho L, Cândido EAF, Gomes MZ. The Effect of Alpinia zerumbet 
Essential Oil on Post-Stroke Muscle Spasticity. Basic Clin Pharmacol Toxicol 2016; 118(1): 58-62.

19. Moher D, Liberati A, Tetzlaff J, Altman DG, Altman D, Antes G, et al. Preferred reporting items for systematic reviews and meta-analyses: The PRISMA statement. PLoS Med. 2009; 6(7).

20. Centre for Evidence-Based Physiotherapy, 2020. Physiotherapy Evidence Database (PEDro). Disponível em: http://www.pedro.org.au/english/downloads/pedro-scale/. (Acessado em 15.07.2020).

21. National Heart, Lung, and Blood Institute, 2020. Development and use of quality assessment tools. Disponível em: http://www. nhlbi. nih. gov/ health- pro/guidelines/ in- develop/ cardiovascularriskreduction/ tools. (Acessado em 15.07.2020).

22. Oliveira VDC, Catarinny D, Carolina Í, Santana DJ, Edilaine M, Ferreira R, et al. Correlation of staticity with funcionality os spastic individuaals after physiotherapy treatment associated with herbal medicine derived from Alpinia Zerumbet. Int J Dev Res 2018; 08(01): 18443-7.

23. Cândido JF, Catarinny D, Carvalho- JN De. Subacute and Chronic Treatment with Herbal Medicine Essential Oil the Alpinia Zerumbet Associated with Kinesiotherapy on Patient with Muscle Spasticity: Cases Series. Int J Res Stud Biosci 2017; 5(10): 1-6.

24. Durigon OFS, Sá CSS, Sitta L V. Validação de um protocolo de avaliação do tono muscular e atividades funcionais para crianças com paralisia cerebral. Rev Neurociências 2004; 12(2): 87-93. 